\title{
Free and forced convection around line sources of heat and heated cylinders in porous media
}

\author{
By VADIM N. KURDYUMOV ${ }^{1,2} \dagger$ AND AMABLE LIÑÁN ${ }^{1}$ \\ ${ }^{1}$ E.T.S. Ingenieros Aeronáuticos, Universidad Politécnica de Madrid, Plaza Cardenal Cisneros 3, \\ 28040 Madrid, Spain \\ ${ }^{2}$ Instituto Tecnológico de Veracruz, Dep. de Metal Mecánica, 91868 Veracruz, Mexico
}

(Received 19 April 1999 and in revised form 6 March 2000)

An analysis is presented for the steady, two-dimensional, free convection around line sources of heat and heated cylinders in unbounded saturated porous media. It is extended to account also for the effects of forced convection. The study is based on the Boussinesq equations, with the velocities calculated using Darcy's law.

The analysis begins with the non-dimensional formulation and numerical solution of the problem of pure free convection around a line source of heat. When this analysis is extended to include the effects of forced convection, two parameters appear in the non-dimensional formulation: the non-dimensional value, $V_{\infty}$, of the free-stream velocity and its angle $\gamma$ of inclination with respect to the vertical. We first describe the asymptotic form of the solution for large and small values of the distance to the source. The far-field description, which is also applicable to the flow around heated cylinders, is needed to facilitate the numerical solution of the problem. It includes a thermal wake, aligned with the free stream, and an outer irrotational flow with a sink and a vortex at the line source. The temperature distribution near the source involves a constant $A_{0}\left(V_{\infty}, \gamma\right)$, to be calculated with the numerical solution of the complete problem, which is used in the evaluation of the heat transfer from heated cylinders when the Rayleigh and Péclet numbers are small compared with unity. In this case we find an inner region where heat conduction is dominant, and an outer region where the cylinder appears as a line source of heat. The asymptotic analysis is complemented with the numerical solution of the general problem for circular cylinders with a wide range of Rayleigh numbers and some representative values of $V_{\infty}$ and $\gamma$. We give correlations for the Nusselt number in the limiting cases of pure free convection and pure forced convection.

\section{Introduction}

This paper is devoted to the description of the planar and steady flow field around heated cylinders in unbounded fluid-saturated porous media, under the combined effects of natural and forced convection. These effects are measured by a Rayleigh number, $R a$, a Péclet number, $P e$, and the angle of inclination $\gamma$ of the free-stream velocity with the vertical. These are the parameters remaining in the non-dimensional formulation of the problem, to be solved numerically, after describing, as we shall do below, the far-field decay of the perturbations to the free stream.

$\dagger$ Author to whom correspondence should be sent: I.T.Ver., Dep. de Metal Mecánica, 91868 Veracruz, Mexico. E-mail: vadim@itver.edu.mx 
Although the problems of heat transfer from heated bodies in porous media under either forced or free convection have received adequate attention in the literature-see, for example the reviews by Gebhart et al. (1988) and Nield \& Bejan (1992) - the analyses devoted to mixed convection around heated cylinders are scarce. They consider, mainly, cases with the flow far from the cylinder parallel to the gravity force. Of this type are the analyses, for large Rayleigh numbers, of Cheng (1982), Huang et al. (1986), and also of Badr \& Pop (1988), who found a recirculating region near the cylinder, when the forced flow was downwards, in the direction of gravity. A notable exception is the work of Vynnycky \& Pop (1997), devoted to the combined effects of free convection and oblique forced flow past cylindrical bodies. They consider a non-uniform surface temperature such that the overall heat lost by the cylinder to the porous medium is zero. With this choice they minimize the difficulties encountered in the description of the far field, needed for a coherent numerical solution of the problem.

We shall pay particular attention to the cases of $P e \ll 1$ and $R a \ll 1$, when the size $l_{h}$ of the heated region surrounding the cylinder is large compared with its radius $a$. In the two-dimensional flow that we consider the rate of heat transfer $q$, per unit length, from the cylinder to the porous medium cannot be calculated using the solution of the pure heat conduction problem, because it diverges far from the cylinder. To calculate $q$ we shall follow the method of matched asymptotic expansions used by Kaplun \& Lagerstrom (1957), for the flow of ordinary fluids around cylinders at small Reynolds numbers, and by Liñán \& Kurdyumov (1998) and Kurdyumov \& Liñán (1999) for the free convection, at low Rayleigh numbers, around heated cylinders and spheres in fluids. If for $R a \ll 1$ and $P e \ll 1$ we look at the flow field with the scale $l_{h}$ of the heated region surrounding the cylinder, this appears as a line source of heat. Therefore, this paper contributes to the literature on the analysis of the flow field around line sources of heat, in $\S 2$ for pure free convection, and in $\S 4$ with the combined effects of forced flow.

For pure free convection around line sources of heat it is possible, in contrast with the case of point sources, to obtain scales, $l_{h}, v_{h}$ and $T_{h}-T_{\infty}$, for the size, velocity and temperature rise in the heated region around the line source. These scales are well defined by the strength of the heat source, $q$, and the independent physico-chemical parameters entering in the flow equations, which become parameter-free when written in non-dimensional form using those scales. The solution, which is not self-similar, must be obtained numerically, after describing its asymptotic far- and near-field forms, for radial distances large and small compared with $l_{h}$.

The corresponding problem for point sources of heat, which for Darcy's law has no characteristic length, was analysed by Bejan (1978). The flow admits a selfsimilar description, depending only on one parameter, a Rayleigh number. Bejan gave asymptotic solutions of the resulting system of ordinary differential equations, for small and large values of a Rayleigh number, and numerical solutions were obtained by Hickox \& Watts (1980). At low Rayleigh numbers, the temperature field is determined by heat conduction everywhere; while for large Rayleigh numbers the heated region becomes a slender plume above the source, where it has its origin.

The existing analyses for line heat sources, see for example Wooding (1963) and Afzal (1985), correspond in fact to the far-field description; there we find a slender thermal plume above the source and an outer irrotational flow due to the entrainment by the plume. They are not valid in the heated region surrounding the source, and cannot be used to calculate the free convection heat transfer from heated cylinders at small Rayleigh numbers. The unsteady flow generated by a constant line heat source, acting only for $t>0$, was analysed by Nield \& White (1982); see also Nield \& Bejan 
(1992, pp. 124-127). The unsteady solution was found as a power series for small values of a parameter corresponding to the time; however, the steady solution cannot be found using this power series.

The pure free convection flow around heated cylinders in unbounded porous media is discussed in $\S 3$. For large values of the Rayleigh number, the temperature changes are confined to a thin boundary layer on the body, which Merkin (1979) analysed for two-dimensional bodies and axisymmetric bodies of arbitrary shape, and to a slender plume above. For $R a \ll 1$ the flow structure and the rate of heat loss $q$ is calculated, in $\S 3$, with the method of matched asymptotic expansions, using the heat source results of $\S 2$. For values of the Rayleigh number of order unity the free convection must be described numerically. The free convection around heated cylinders was calculated with finite difference methods, for Rayleigh numbers up to 400, by Ingham $\&$ Pop (1987). They used the heuristic patching method of Mahony (1957) to obtain a correlation of the heat transfer for small Rayleigh numbers. Numerical solutions for the natural convection around heated cylinders, buried in semi-infinite and bounded porous media, were presented by Farouk \& Shayer (1988) and by Himasekhar \& Bau (1988). In $\S 3$, we complement the existing asymptotic analyses for large Rayleigh numbers, and our asymptotic analysis for small values, with additional numerical calculations to generate a correlation between the Nusselt and Rayleigh numbers. We shall also show in $\S 3.3$ how to account for arbitrary, non-vertical, inclinations of the heated cylinders.

In $\S \S 4$ and 5 we deal with the combined effects of forced and free convection in the flow around line sources of heat and heated cylinders. Without buoyancy forces the forced flow in porous media around cylindrical bodies is irrotational and without circulation. The temperature field associated with this forced flow around a heated cylinder includes a thermal wake, determined by $q$ and the free-stream velocity $U_{\infty}$. However, if we retain the effects of the buoyancy forces the flow field changes drastically. In particular, a circulation is established in the far field, because a jump in pressure across the wake is required to balance those forces. This circulation involves a much slower decay of the velocity perturbations than those associated with the dipole corresponding to the basic irrotational flow around the cylinder.

We analyse, in $\S 4$, the combined forced and free convection in a porous medium around a horizontal line source of heat, when the velocity $U_{\infty}$ forms an angle $\gamma$ with the vertical. The non-dimensional form of the problem, based on the pure freeconvection scales, involves two parameters, $\gamma$ and the ratio, $V_{\infty}$, of $U_{\infty}$ to the free convection characteristic velocity $v_{h}$. Typical cases are described numerically, taking into account the asymptotic form of the solution for small and large distances from the line source. For $r / l_{h} \ll 1$, the natural convection is dominant, and the solution has the form of the pure free convection case. The analytical far-field description includes perturbations of the external flow induced by the buoyancy forces; these perturbations are stronger in a plume or thermal wake, which is aligned with the forcing flow, than in the outer isothermal irrotational flow, including a sink and a vortex at the origin.

The effects of buoyancy on the forced flow around heated cylinders are described in $\S 5$. The analysis involves the Rayleigh number, $R a$, in addition to the parameters $\gamma$ and $V_{\infty}$. We have carried out a numerical analysis of the flow for various representative values of $R a, V_{\infty}$ and $\gamma$, and we have used again the technique of matched asymptotic expansions to describe the solution for small values of $R a$, with $V_{\infty}$ of order unity.

Wesseling (1975) analysed the corresponding problem of the combined effects of forced flow and free convection around point and line sources of heat in homogeneous 
fluids. The relative importance of the two effects was measured, as in our analysis, by the angle of inclination and a parameter, similar to our $V_{\infty}$. He could solve the problem for the point source for values of $V_{\infty} \gg 1$, but he could not find a solution for the line source. He concluded that, even when $V_{\infty} \gg 1$, the perturbations due to free convection are too strong in the far field to allow for a consistent, steady and two-dimensional far-field representation, and that the solution of the problem does not exist for arbitrary angles between the forced flow and gravity. The far field in the ordinary flow around unheated bodies is well known; see, for example, the description in Landau \& Lifshitz (1987) for three-dimensional bodies in laminar subsonic flow in terms of their lift and drag. See also the more general far-field description by Chang (1961) and by Lagerstrom (1996). We have tried, but, like Wesseling, could not find an equivalent description for the far field in the forced fluid flow around heated cylinders when the effects of the buoyancy forces are retained, even though we did not encounter these difficulties, as we shall see below, for the solution of the equivalent problem in porous media. We agree with Wesseling's conjecture of non-existence of steady two-dimensional solutions for the forced ordinary fluid flow around heated cylinders in the presence of gravity forces, unless gravity is parallel to the free-stream velocity.

\section{Free convection due to a line source of heat}

\subsection{Formulation}

Consider the steady free convection flow induced by a horizontal line source of heat, of intensity $q$, embedded in an unbounded porous medium of constant permeability $K$, with the velocity determined, through the linear Darcy's law, by the pressure and buoyancy forces due to the gravity $\boldsymbol{g}$; the fluid far from the source is stagnant with a uniform temperature $T_{\infty}$. We shall also assume that the fluid and solid in the porous medium have locally the same temperature. The density changes of the fluid are considered to be small enough so that the Boussinesq approximation is applicable.

The velocity $\boldsymbol{v}$, temperature $T$ and variation of pressure $p^{\prime}$ from its hydrostatic value satisfy, see Gebhart et al. (1988) or Nield \& Bejan (1992), the equations

$$
\begin{gathered}
\nabla \cdot \boldsymbol{v}=0, \\
\frac{\mu_{f}}{K} \boldsymbol{v}=-\nabla p^{\prime}-\boldsymbol{g} \rho_{f} \beta\left(T-T_{\infty}\right), \\
\boldsymbol{v} \cdot \nabla T=\alpha \Delta T,
\end{gathered}
$$

where $\mu_{f}$ is the viscosity, $\rho_{f}$ is the density, and $\beta$ is the coefficient of volumetric expansion of the fluid; $\alpha$ is the effective thermal diffusivity of the saturated porous medium. These equations must be solved with the boundary conditions

$$
\boldsymbol{v} \rightarrow 0, \quad p^{\prime} K / \mu_{f} \rightarrow 0, \quad \text { and } \quad T-T_{\infty} \rightarrow 0 \quad \text { at } \quad r \rightarrow \infty .
$$

The effect of the line heat source is taken into account by requiring that

$$
\lim _{r \rightarrow 0} \lambda \int_{0}^{2 \pi} r \frac{\partial T}{\partial r} \mathrm{~d} \varphi=-q
$$

where $r$ is the radial distance to the line source, and $\lambda$ is the effective thermal conductivity of the fluid-saturated porous medium.

The two independent parameters, $K \rho_{f} \beta g / \mu_{f}$ and $\alpha$, appearing in the equations, and 
$q / \lambda$, appearing in the boundary condition (2.5), define the following scales:

$$
l_{h}=\frac{\mu_{f} \alpha \lambda}{K \rho_{f} g \beta q}, \quad v_{h}=\frac{K \rho_{f} \beta g q}{\mu_{f} \lambda}, \quad T_{h}-T_{\infty}=\frac{q}{\lambda},
$$

corresponding to the characteristic values of the size, $l_{h}$, velocity, $v_{h}$, and temperature rise above the ambient in the heated region around the source. These scales can also be determined by means of order-of-magnitude estimates of the terms appearing in (2.2)-(2.5). The characteristic temperature rise, $T_{h}-T_{\infty}=q / \lambda$, results from the source condition (2.5); if the first and second terms in equation (2.2) are of the same order, $\mu_{f} v_{h} / K=g \beta \rho_{f}\left(T_{h}-T_{\infty}\right)$; and, from the balance of convection and conduction in (2.3), we obtain $l_{h} v_{h} / \alpha=1$, i.e. the Péclet number based on $l_{h}$ and $v_{h}$ is 1 .

When the fluid is a gas $\beta=1 / T_{\infty}$, and for the Boussinesq approximation to be applicable in the heated region $r \sim l_{h},\left(T_{h}-T_{\infty}\right) / T_{\infty}=q / \lambda T_{\infty}$ must be small compared with unity. For the Darcy's law to be applicable $\rho_{f} \sqrt{K} v_{h} / \mu_{f} \ll 1$.

Using $l_{h}$ and $v_{h}$ as scales for the spatial coordinates and for the velocity, and defining $\theta=\left(T-T_{\infty}\right) /\left(T_{h}-T_{\infty}\right)$ as the non-dimensional temperature rise, the system of equations (2.1)-(2.3), when written using the cylindrical coordinates $r$, distance to the line source, and $\varphi$, angle with the vertical direction, takes the form

$$
\begin{gathered}
\frac{\partial^{2} \psi}{\partial r^{2}}+\frac{1}{r} \frac{\partial \psi}{\partial r}+\frac{1}{r^{2}} \frac{\partial^{2} \psi}{\partial \varphi^{2}}=\frac{\partial \theta}{\partial r} \sin \varphi+\frac{1}{r} \frac{\partial \theta}{\partial \varphi} \cos \varphi, \\
v_{r} \frac{\partial \theta}{\partial r}+\frac{v_{\varphi}}{r} \frac{\partial \theta}{\partial \varphi}=\frac{\partial^{2} \theta}{\partial r^{2}}+\frac{1}{r} \frac{\partial \theta}{\partial r}+\frac{1}{r^{2}} \frac{\partial^{2} \theta}{\partial \varphi^{2}} .
\end{gathered}
$$

The stream function $\psi$ is defined by $v_{r}=r^{-1} \partial \psi / \partial \varphi$ and $v_{\varphi}=-\partial \psi / \partial r$.

The equations are to be solved, for $r>0$, subject to the condition

$$
\lim _{r \rightarrow 0} \int_{0}^{2 \pi} r \frac{\partial \theta}{\partial r} \mathrm{~d} \varphi=-1
$$

associated with a unit line source of heat at $r=0$. The boundary conditions far from the source require

$$
\theta \rightarrow 0, \quad \boldsymbol{v} \rightarrow 0, \quad \text { as } \quad r \rightarrow \infty .
$$

Although $v$ tends to zero as $r \rightarrow \infty$, the decay is not fast enough to prevent the stream function, $\psi$, from growing to infinity.

The flow and temperature fields are symmetrical with respect to the vertical plane containing the source, so that the equations need to be solved only for $0<\varphi<\pi$ using the following symmetry conditions:

$$
\psi=\frac{\partial \theta}{\partial \varphi}=0 \quad \text { at } \quad \varphi=0 \quad \text { and } \quad \varphi=\pi .
$$

No parameter is left in the system (2.7)-(2.10) of equations and boundary conditions describing the natural convection around a line heat source in a porous medium. The solution of the problem must be obtained numerically, and for this we need analytical descriptions for the field far and near the source.

\subsection{Asymptotic description for large and small $r$}

The description for large values of $r$ includes a buoyant plume above the source, where $\psi$ and $\theta$ are given by inverse coordinate expansions in $r$ :

$$
\psi=r^{1 / 3} f_{0}(\zeta)+f_{1}(\zeta)+\cdots, \quad \theta=r^{-1 / 3} g_{0}(\zeta)+r^{-2 / 3} g_{1}(\zeta)+\cdots,
$$


where $\zeta=r^{1 / 3} \varphi$, the self-similar variable, is of order unity in the plume, whose thickness is of order $r^{2 / 3}$, while the temperature and vertical velocity in the plume are of order $r^{-1 / 3}$. The first terms correspond to the self-similar solution of the boundary layer form of the equations, associated with the known constant value of the vertical convective energy flux. $\uparrow$ To determine the unknown functions we substitute the series (2.12) in the equations (2.7) and (2.8) and equate coefficients of like power of $r$. The corresponding equations were obtained by Wooding (1963), for $f_{0}$ and $g_{0}$, and by Afzal (1985), for $f_{1}$ and $g_{1}$, and solved using the appropriate matching conditions with the outer flow, together with the integral conditions

$$
\int_{0}^{\infty} f_{0}^{\prime} g_{0} \mathrm{~d} \zeta=\frac{1}{2}, \quad \int_{0}^{\infty}\left(f_{0}^{\prime} g_{1}+g_{0} f_{1}^{\prime}\right) \mathrm{d} \zeta=0
$$

stating that the total convective heat flux in the plume is equal to the strength of the source.

For large $r$, outside the thermal plume $\theta=0$, and the flow, due to entrainment by the plume, is irrotational, with the stream function satisfying the Laplace equation, $\Delta \psi=0$. The asymptotic form of the solution matching with the expansion (2.12), for the plume, is given by

$$
\psi=A r^{1 / 3} \sin ((\pi-\varphi) / 3)+B(\pi-\varphi)+\cdots, \quad 0<\varphi<\pi,
$$

with the constants $A$ and $B$ chosen so that (2.14) matches for $\varphi \rightarrow 0$ with (2.12). The velocities resulting from (2.14) decrease with $r$ like $r^{-2 / 3}$.

The results, obtained by Wooding (1963) and by Afzal (1985), take the form

$$
\begin{gathered}
f_{0}=f_{\infty} \tanh s, \\
g_{0}=\left(f_{\infty}^{2} / 6\right) \operatorname{sech}^{2} s, \\
f_{1}=-(2 s / \sqrt{3})+\left(1+s(\exp s) \operatorname{sech}^{2} s-\operatorname{sech} s\right) / \sqrt{3}, \\
g_{1}=\left(\sqrt{3} f_{\infty} / 9\right)(1-s \tanh s) \operatorname{sech}^{2} s,
\end{gathered}
$$

where $f_{\infty}=(9 / 2)^{1 / 3}$ and $s=f_{\infty} \zeta / 6$. Then, $A=36^{1 / 3} / \sqrt{3}$ and $B=\sqrt{3} / 3 \pi$.

The far flow field can be represented, for $r \gg 1$ and $0<\varphi<\pi$, by the composite expansion

$$
\psi_{c}=\left\{r^{1 / 3} f_{0}(\zeta)+A r^{1 / 3} \sin \left(\frac{\pi-\varphi}{3}\right)-\frac{\sqrt{3}}{2} A r^{1 / 3}\right\}+\left\{f_{1}(\zeta)-B \varphi+\frac{A}{6} \zeta\right\}+\cdots
$$

based on the plume solution (2.12) and the irrotational flow (2.14). The temperature field for $r \gg 1$ can be represented by the plume expansion (2.12), because $\theta$ tends to zero when $\varphi \gg r^{-1 / 3}$. Notice that no unknown constants are left in the first two terms of the far-field expansion. If we needed to retain higher-order terms, associated with the eigenfunctions of the boundary layer form of the equations, the unknown multiplying constants could only be obtained with the full numerical solution of the problem.

$\dagger$ There are other terms in the expansions, which are eigenfunction solutions of the boundary layer approximation of the equations (2.7)-(2.8), not explicitly included in (2.12) because they show a faster decay to zero with $r$; see Van Dyke (1975) for a discussion of the expected form of the coordinate expansions. These asymptotic expansions are often considered in the literature as large Rayleigh number expansions, because an artificial length $L \gg l_{h}$ is introduced and $r$ considered $\sim L$. 
For small values of $r$, the temperature and stream function will be described with coordinate expansions of the form

$$
\begin{aligned}
& 2 \pi \theta=-\ln r+A_{0}+A_{1} r \cos \varphi+\cdots, \\
& \psi=\left(-\frac{1}{4 \pi} r \ln r+B_{0} r\right) \sin \varphi+\cdots,
\end{aligned}
$$

where the terms shown in (2.20), which satisfy the condition (2.9), are solutions of the Laplace equation, or a simplified form of (2.8) when the convective terms are neglected. The terms appearing in the expansion (2.21) are solutions of (2.7) with the right-hand side evaluated using (2.20). The first of these terms, also showing a logarithmic singularity of the velocity at $r \rightarrow 0$, is due to the buoyancy force. More singular solutions have been eliminated from (2.20) and (2.21). The constants that appear in these expansions must be obtained as part of the numerical solution of the complete line source problem. The constants $A_{0}$ and $A_{1}$ determine, respectively, the temperature level near the source and a vertical temperature gradient.

\subsection{Numerical solution for values of $r \sim 1$}

Due to the singular character of the solution of the equations (2.7) and (2.8) for $r \rightarrow 0$ and for $r \rightarrow \infty$, we shall solve numerically these equations in the finite domain $r_{\min }<r<r_{\max }$. We can take into account (2.20) and (2.21) to write the boundary conditions for the temperature and stream function at $r \rightarrow 0$ in the form

$$
r \frac{\partial \theta}{\partial r}+\frac{1}{2 \pi}=r \frac{\partial \psi}{\partial r}-\psi+\frac{r \sin \varphi}{4 \pi}=0 .
$$

This weak form of the inner boundary conditions, which we impose at $r=r_{\min } \ll 1$, does not involve the unknown constants appearing in (2.20) and (2.21).

At a finite outer boundary, at $r=r_{\max } \gg 1$, two types of boundary conditions have been used. In the first type the stream function and temperature at $r=r_{\max }$ take the values given by the composite expansion (2.19) and by (2.12). The second type of conditions is based on the division, according to (2.19), of the boundary $r=r_{\max }$ into an inflow interval, $\varphi_{*}<\varphi<\pi$, where $v_{r}$ is negative, and an outflow interval, $0<\varphi<\varphi_{*}$, with positive radial velocity. The separation angle, $\varphi_{*}$, depends on the value of $r_{\max }$. At the inflow boundary we used (2.19) and $\theta=0$, while at the outflow boundary we used the following mild boundary conditions:

$$
\frac{\partial^{2} \psi}{\partial r^{2}}=\frac{\partial \theta}{\partial r}=0
$$

No significant differences have been observed in the stream function and temperature distributions, in the main part of computational domain, when using the two types of boundary conditions, aside from small differences near the outflow boundary.

The governing equations (2.7) and (2.8), written in terms of $\eta=\ln r$ to improve the accuracy of the numerical solution at small values of $r$, were solved using a secondorder, three-point, finite-difference approximation for the first and second derivatives. To obtain the stationary distributions of all variables a pseudo-unsteady form of the governing equations was used. We consider that the stationary distribution has been reached when $\max _{i, j}\left|f_{i, j}-\hat{f}_{i, j}\right|<10^{-9}$, where $f$ and $\hat{f}$ are the values of the temperature or the stream function at the current and previous levels. Calculations were carried out using $101 \times 101$ and $201 \times 201$ points to test the grid independence, and with different values of $r_{\min }$ and $r_{\max }$ to ensure independence from the computational 


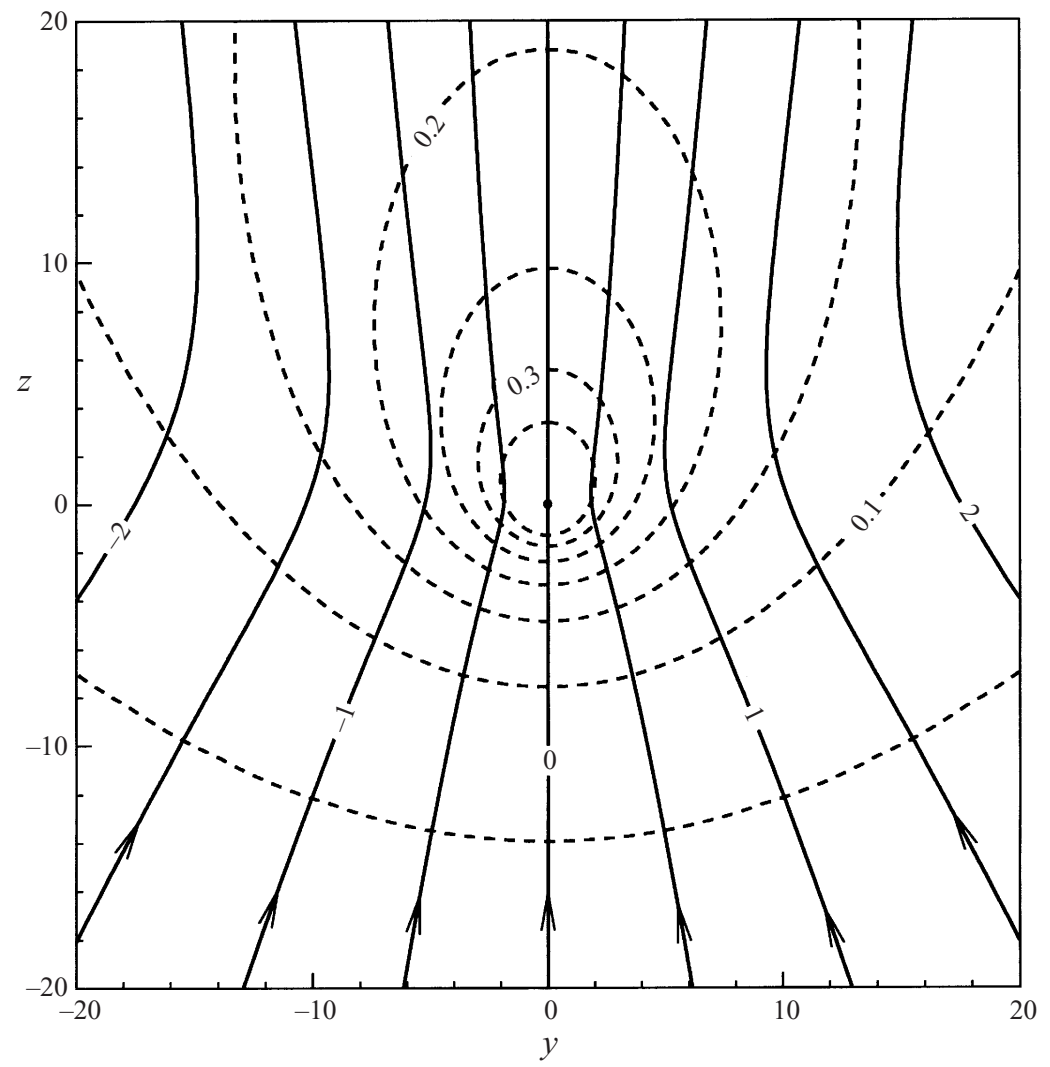

FIGURE 1. Pure free convection around a line source of heat: streamlines (solid lines) and isotherms (dashed lines).

domain. For values of $r_{\min }$ below $10^{-2}$ and $r_{\max }$ above $10^{2}$ no significant changes in the temperature distribution and flow fields were observed.

Shown in figure 1, with dashed and solid lines, are the isotherms and streamlines of the pure free convection due to a horizontal line source of heat. The vertical velocity and temperature distributions in the centre-plane, $y=0$, are shown in figure 2 with solid and dashed lines; for comparison we also give the results provided by the first two terms of the far-field expansion. The values of the constants $A_{0}$ and $B_{0}$, appearing in the small-r description (2.20) and (2.21), have been found to be 2.71 and 0.317 , respectively.

\section{Free convection heat transfer from heated circular cylinders in porous media}

We shall begin with the analysis, in $\S \S 3.1$ and 3.2, of the free convection around horizontal heated cylinders. It will be extended to inclined, non-vertical, cylinders in $\S 3.3$.

\subsection{Formulation and numerical description for $R a \sim 1$}

Because the far field is determined by $q$, the rate of heat transferred per unit length of the cylinder to the porous medium, in order to facilitate the numerical solution we shall pose the problem as to find the uniform value $T_{w}$ of the surface temperature leading to a given value of $q$. With the additional conditions (2.4) the system of 


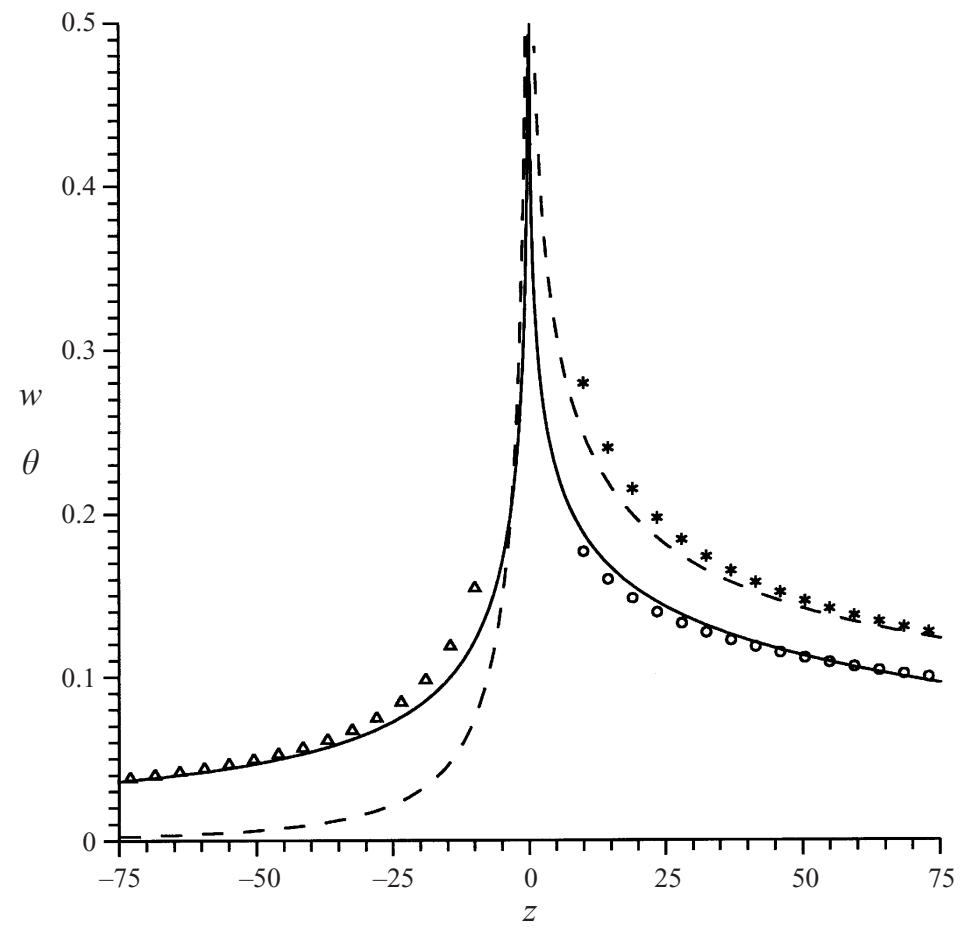

FIGURE 2. Pure free convection: computed temperature distribution (dashed lines) and vertical velocity (solid lines) in the vertical plane of the line source. Asymptotic descriptions are also given (with triangles) for the temperature, $\theta=0.4543 z^{-1 / 3}+0.3177 z^{-2 / 3}$, velocity above the line source, $w=0.4543 z^{-1 / 3}-0.1589 z^{-2 / 3}$ (with circles), and below the source, $w=0.6355(-z)^{-2 / 3}+0.1838(-z)^{-1}$ (with stars)

equations (2.1)-(2.3) must be solved in the domain of the porous medium, outside the cylinder. The problem, for a circular cylinder of radius $a$, will be formulated in non-dimensional form using the scales (2.6). Then, we are left with the system of equations (2.7)-(2.8), to be solved with the boundary conditions (2.10), at $r \rightarrow \infty$, and

$$
\psi=0, \quad \theta=\theta_{w}, \quad \text { at } \quad r=\varepsilon=a / l_{h},
$$

the cylinder surface. The surface temperature $\theta_{w}$, considered uniform, must be determined as a function of $\varepsilon$, the non-dimensional radius of the cylinder, to ensure that

$$
\int_{0}^{2 \pi} r \frac{\partial \theta}{\partial r} \mathrm{~d} \varphi=-1 \quad \text { at } \quad r=\varepsilon .
$$

The Nusselt and Rayleigh numbers, defined by

$$
N u=\frac{q}{2 \pi \lambda\left(T_{w}-T_{\infty}\right)}, \quad R a=\frac{K \rho_{f} \beta g\left(T_{w}-T_{\infty}\right) a}{\alpha \mu_{f}},
$$

can be written in terms of $\varepsilon$, the only parameter left in the problem, as

$$
N u=1 / 2 \pi \theta_{w}(\varepsilon) \text { and } R a=\varepsilon \theta_{w}(\varepsilon)
$$

giving a parametric representation of $N u(R a)$.

The numerical technique described in $\S 2$ was used to solve equations (2.7)-(2.8), in the domain $\varepsilon<r<r_{\max }$, using with the far-field behaviour given by (2.12) and 
(2.19), and the conditions (3.1) and (3.2) at $r=\varepsilon$. The calculated values of the Nusselt number are shown in figure 4 with circles for a wide range of $R a$, down to $10^{-2}$.

3.2. Heat transfer at $R a \ll 1$, and correlation of $N u(R a)$ for all values of $R a$ We can anticipate from (3.4) that $R a \ll 1$ leads to $\varepsilon \ll 1$. This implies that the flow and temperature fields can be divided into two regions with disparate scales $l_{h}$ and $a$. In the outer region, scaled with $l_{h}$, the hot cylinder acts, in a first approximation, as a pure line source of heat. In the inner region, scaled with $a$, the effects of convection are negligible, in a first approximation.

The inner solution for $r / \varepsilon \sim 1$ is given in dimensionless form for $\varepsilon \ll 1$ by the expansions

$$
\begin{gathered}
2 \pi \theta=2 \pi \theta_{w}-\ln (r / \varepsilon)+\cdots \\
\psi=\left\{B r-\frac{1}{4 \pi} r \ln r-\left(B \varepsilon-\frac{1}{4 \pi} \varepsilon \ln \varepsilon\right) \frac{\varepsilon}{r}\right\} \sin \varphi+\cdots .
\end{gathered}
$$

The requirement that the distributions (2.20) and (2.21) coincide with (3.5) and (3.6) in the intermediate region $\varepsilon \ll r \ll 1$ leads to $B=B_{0}$, and the relation

$$
2 \pi \theta_{w}+\ln \varepsilon=A_{0}
$$

between the surface temperature of the cylinder and $\varepsilon$. Here $B_{0}=0.317$ and $A_{0}=2.71$, are taken from the results of $\S 2.3$. Taking into account (3.4), the following relation is obtained between the Nusselt and Rayleigh numbers:

$$
N u^{-1}=A_{0}-\ln (2 \pi N u R a) .
$$

Based on the asymptotic relation (3.8) for $R a \ll 1$, and our numerical calculations, we propose a correlation between $R a$ and $N u$ for all values of $R a$ not invalidating the linear Darcy's law of the form

$$
R a=\frac{1}{2 \pi N u} \exp \left(2.71-\frac{1}{N u}\right) F(N u)
$$

with the coefficients in the function $F$

$$
F=1+a_{1} N u^{1 / 2}+a_{2} N u^{1}+a_{3} N u^{3 / 2}+a_{4} N u^{2}+a_{5} N u^{3}
$$

chosen to ensure that (3.9) coincides with (3.8) for $R a \rightarrow 0$ (when $N u \rightarrow 0$ ). We have also taken into account the relation $N u=b R a^{1 / 2}$, with $b=0.3995$, obtained by Merkin (1979) in his analysis of the thermal boundary layer for large Rayleigh numbers (when $N u \gg 1$ ); therefore $a_{5}=2 \pi b^{-2} \exp (-2.71)=2.592$. Finally, the values of the remaining coefficients in (3.10), $a_{1}=1.088, a_{2}=-6.581, a_{3}=11.31$ and $a_{4}=-4.866$, are chosen so as to correlate the results, shown with circles in figure 3 , of our numerical calculations. The correlation (3.9) is shown in figure 3 with a solid line; also shown with dashed lines are the asymptotic relation (3.8), for $R a \ll 1$, and the correlation proposed for $R a \gg 1$ by Ingham \& Pop (1987)

$$
N u=0.3995 R a^{1 / 2}+0.78-1.9 R a^{-1 / 2} .
$$

3.3. Free convection around inclined, non-vertical, line sources of heat and cylinders The effects of the inclination with respect to the horizontal of an infinitely long line source of heat or hot cylinder can be easily taken into account. We shall use a coordinate system with $x$ (positive downwards) in the direction of the line source 


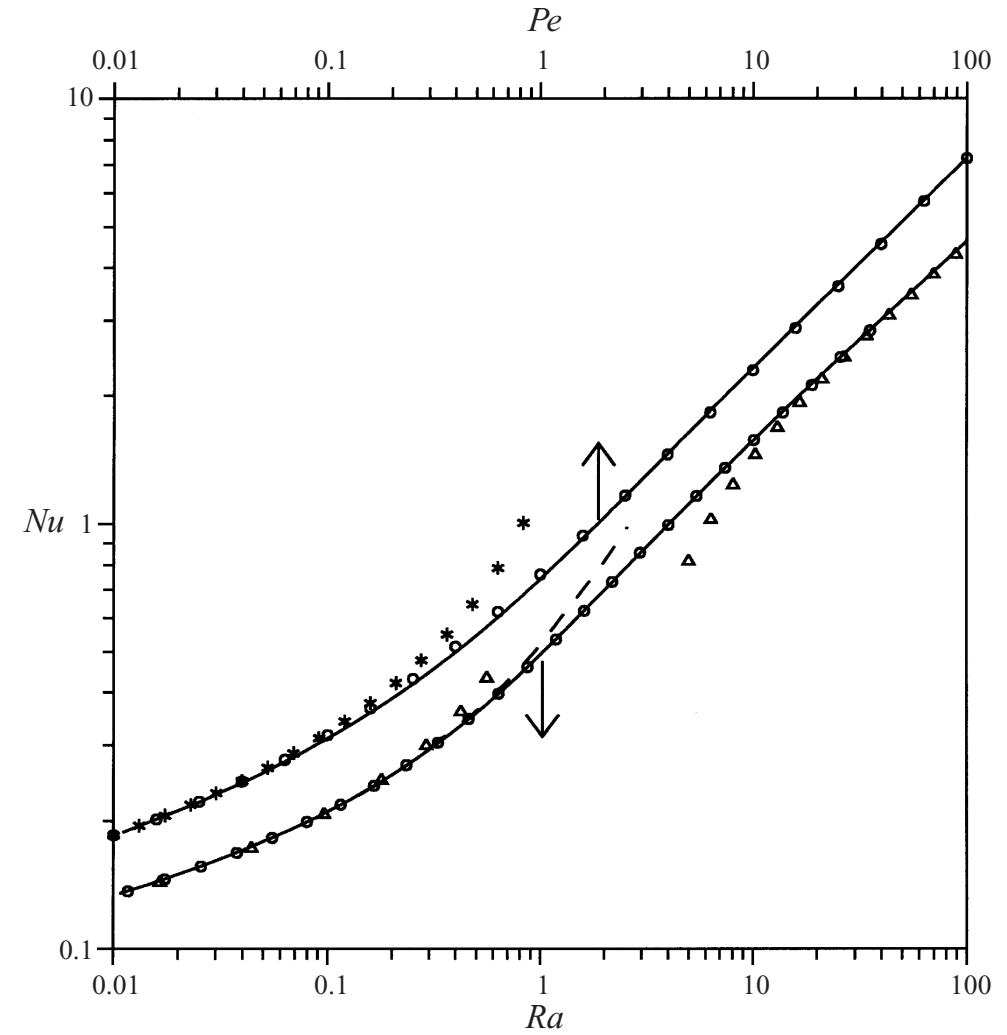

FIGURE 3. Average Nusselt number as a function of the Rayleigh number for pure free convection, and of the Péclet number for pure forced convection; numerical results are shown with circles and correlation formulae (3.9) and (5.7) with solid lines. Triangles: asymptotic relations (3.8) and (3.11) for small and large values of Ra. Stars: asymptotic description (5.6) for small values of $P e$. Dashed line: average Nusselt number in terms of the Rayleigh number for the case of combined convection with $V_{\infty}=0.1$ and $\gamma=\pi / 2$.

or the cylinder and two coordinates, $y$ and $z$ (positive upwards), to characterize the points in planes normal to this direction, chosen so that the components of the gravity acceleration with respect to this coordinate system are $\left(g_{l}, 0,-g_{n}\right)$. Due to the translation invariance, with respect to the coordinate $x$, of the equations and boundary conditions, the temperature and the velocity components are only functions of the coordinates $y$ and $z$. Hence, if we replace $g$ by $g_{n}$ in the definition (2.6) of the velocity and length scales, $v_{h}$ and $l_{h}$, the temperature, the pressure and the transverse velocity components $u$ and $v$ are functions only of $y$ and $z$, given by the previous analysis for horizontal line sources and hot cylinders. The longitudinal velocity component $u$, when also measured with the scale $v_{h}$, is given by $u / \theta=-g_{l} / g_{n}=-\tan \phi$, where $\phi$ is the angle of the inclination of the line source or cylinder with respect to the horizontal. No steady solution exists for vertical cylinders without edges, for which $g_{n}=0$.

\section{A horizontal line source of heat in a uniform stream}

4.1. Formulation and near- and far-field descriptions

Consider a horizontal line source of heat in a porous medium, when far from the source the fluid has a velocity $U_{\infty}$ and temperature $T_{\infty}$. For the description of the 


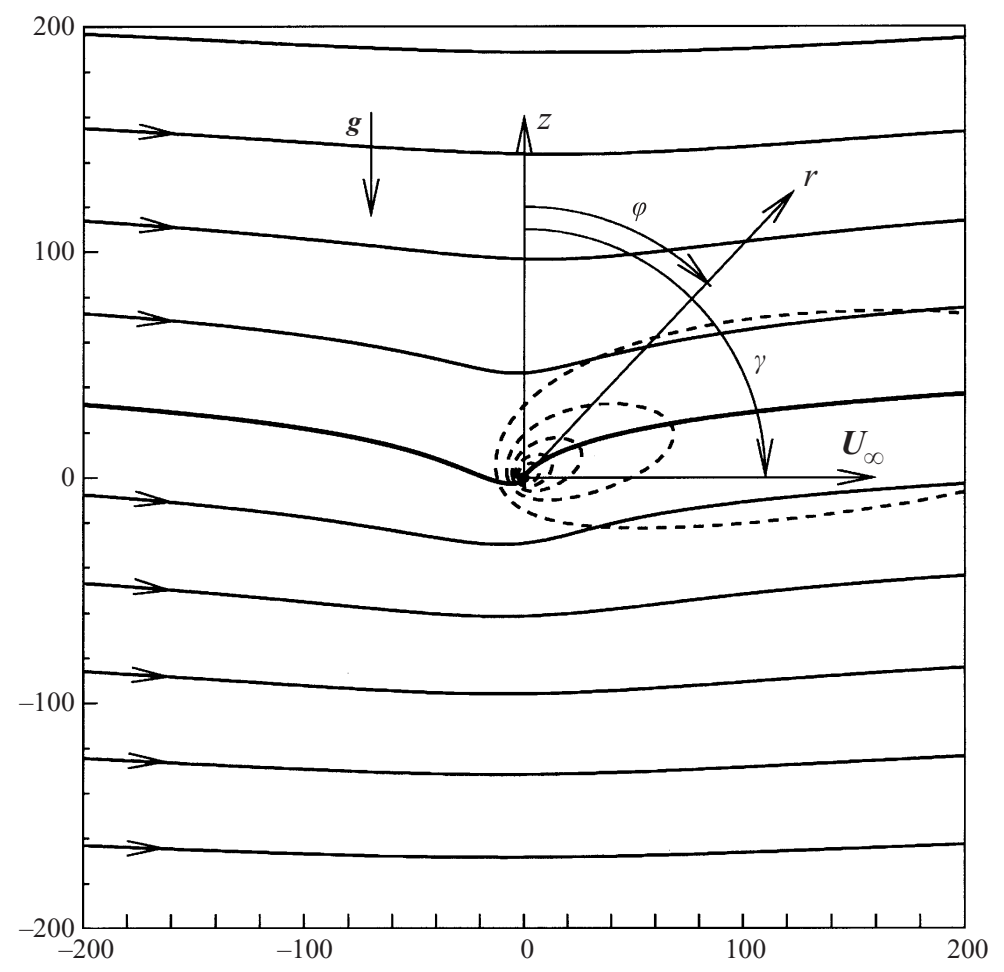

FIGURE 4. System of coordinates and definition of $\gamma$ for forced and natural convection around a line source of heat. The computed streamlines and isotherms correspond to the case $V_{\infty}=0.1$ and $\gamma=\pi / 2 . \psi$ is given with solid lines at intervals $4 ; \theta$ is given with dashed lines at 0.05 intervals between 0.05 and 0.3 .

flow we shall use the coordinate system shown in figure 4. The angle $\gamma$ characterizes the direction of the forced convection; when $\gamma=0$ the forced flow is vertical and upwards.

With the variables measured with the scales (2.6), corresponding to free convection, the governing equations (2.7)-(2.8) have to be solved with the conditions (2.9), at $r \rightarrow 0$, and

$$
\psi / r \rightarrow V_{\infty} \sin (\varphi-\gamma) \text { and } \theta \rightarrow 0, \quad \text { at } \quad r \rightarrow \infty,
$$

and the requirement that $\psi$ and $\theta$ are $2 \pi$-periodic in $\varphi$; we can choose $\psi$ to be zero at $r=0$. The non-dimensional value, $V_{\infty}=U_{\infty} / v_{h}$, of the uniform velocity of the free stream and the angle $\gamma$ are the only parameters remaining in the non-dimensional formulation of the problem.

For the numerical solution we shall begin with the asymptotic representation of the solution for $r \ll 1$ and $r \gg 1$. In the vicinity of the source, for $r \ll 1$, heat conduction and the flow due to free convection are dominant, being responsible for the singular behaviour of $\theta$ and $\psi$; the leading terms are given by

$$
\begin{gathered}
2 \pi \theta=-\ln r+A_{0}+A_{1} r \cos \left(\varphi-\gamma_{1}\right)+\cdots, \\
\psi=\left(-\frac{1}{4 \pi} r \ln r\right) \sin \varphi+B_{0} r \sin \left(\varphi-\gamma_{2}\right)+\cdots,
\end{gathered}
$$


where the constants $A_{0}, A_{1}, B_{0}, \gamma_{1}$ and $\gamma_{2}$ can only be calculated, as functions of $V_{\infty}$ and $\gamma$, with the numerical solution of the problem.

Because in pure free convection in porous media the velocity in the plume decreases like $r^{-1 / 3}$, we can anticipate that the forced convection will dominate the far field; so that, as seen in figure 4 , the plume will be aligned, at $r \gg 1$, with the free-stream velocity, as a thermal wake. In this thermal wake, for large values of $r$, the longitudinal velocity is in a first approximation $V_{\infty}$, and the temperature satisfies the equation

$$
V_{\infty} \frac{\partial \theta}{\partial l}=\frac{\partial^{2} \theta}{\partial n^{2}}
$$

written with coordinates $l$ and $n$ parallel and normal to the wake, both measured with $l_{h}$. The coordinates $l$ and $n$ can be approximated by $r$ and $r(\varphi-\gamma)$ for large $r$, where the solution of (4.4) satisfying the conditions $\theta=0$ at $n \rightarrow \pm \infty$ and the condition

$$
\int_{-\infty}^{+\infty} V_{\infty} \theta \mathrm{d} n=1
$$

of global thermal energy conservation is self-similar, and given by

$$
\theta \sqrt{r}=G_{0}(\xi)=\exp \left(-V_{\infty} \xi^{2} / 4\right) / 2 \sqrt{V_{\infty} \pi},
$$

in terms of the similarity variable $\xi=n / \sqrt{l}=\sqrt{r}(\varphi-\gamma)$, of order unity within the thermal wake, whose thickness grows like $\sqrt{r}$. When writing (4.6), we implicitly assume that the peak temperature lies at $\xi=\xi_{m}=0$ even though $\xi_{m}$ is not determined in a first approximation. Although the value of $\xi_{m}$ would locate more precisely the thermal wake position, it does not influence the outer irrotational velocity perturbations.

The momentum equation in the direction of the wake takes the simplified form

$$
u_{l}^{\prime}=\theta \cos \gamma
$$

allowing us to calculate the longitudinal component, $u_{l}^{\prime}$, of the velocity perturbations. When writing this equation we have neglected, because it is of higher order, the term representing the effect of the changes in pressure, $p^{\prime \prime}$, due to the buoyancy forces. Notice that associated with $u_{l}^{\prime}$, there is an outflow in the wake, with a volumetric flux, given by

$$
\int_{-\infty}^{+\infty} u_{l}^{\prime} \mathrm{d} n=\cos \gamma / V_{\infty},
$$

which is independent of $r$. This must be compensated by an outer irrotational flow, represented by a sink of the same strength at the line source.

The buoyancy forces normal to the wake create a pressure jump in $p^{\prime \prime}$, which can be calculated using the simplified form

$$
0=-\frac{\partial p^{\prime \prime}}{\partial n}-\theta \sin \gamma
$$

of the momentum equation normal to the wake.

By integrating (4.9) across the wake we obtain the pressure jump

$$
\left[p^{\prime \prime}\right]=-\int_{-\infty}^{+\infty} \theta \sin \gamma \mathrm{d} n=-\sin \gamma / V_{\infty},
$$

which is independent of $r$, and leads to an outer irrotational motion

$$
v_{r}^{\prime}=0, \quad v_{\varphi}^{\prime}=-\frac{\sin \gamma}{2 \pi V_{\infty}} \frac{1}{r}
$$

corresponding to a vortex at the origin, with circulation $\sin \gamma / V_{\infty}$. 
The previous results can be summarized now as the first terms of an asymptotic representation, for large values of $r$, of the solution of equations (2.7) and (2.8) for $\theta$ and $\psi$. This is given, within the thermal wake, by

$$
\theta=r^{-1 / 2} G_{0}(\xi)+\cdots, \quad \psi=r^{1 / 2} V_{\infty} \xi+D_{1} \ln r+F_{1}(\xi)+C_{1}+\cdots,
$$

where $F_{1}=\cos \gamma \operatorname{erf}\left(\sqrt{V_{\infty}} \xi^{2} / 4\right) /\left(2 \pi V_{\infty}\right)$, and $\xi=r^{1 / 2}(\varphi-\gamma)$ is of order unity. In the outer region

$$
\theta=0, \quad \psi=V_{\infty} r \sin (\varphi-\gamma)+D_{1} \ln r-B_{1}(\varphi-\gamma-\pi)+C_{1}+\cdots
$$

with $D_{1}=\sin \gamma / 2 \pi V_{\infty}$ and $B_{1}=\cos \gamma / 2 \pi V_{\infty} ; C_{1}$ can only be obtained from the numerical solution of the complete problem.

Then, the leading terms of the composite expansion, valid for $r \gg 1$ and $\gamma-\pi<$ $\varphi<\gamma+\pi$, are

$$
\psi_{c}=V_{\infty} r \sin (\varphi-\gamma)-\frac{\cos \gamma}{2 V_{\infty}}\left(\frac{\varphi-\gamma}{\pi}-\operatorname{erf}\left(\frac{\sqrt{V_{\infty} \xi}}{2}\right)\right)+\frac{\sin \gamma}{2 \pi V_{\infty}} \ln r+C_{1}
$$

for the stream function $\psi$, and (4.6) for $\theta$.

\subsection{Numerical solution for the line heat source problem}

In the numerical solution of the problem we shall use the asymptotic representation of $\psi$ and $\theta$ given above. Hence, at $r=r_{\min } \ll 1$, we can use the inner boundary conditions (2.22); and at a finite outer boundary, $r=r_{\max } \gg 1$, we shall use (4.12) and (4.14). We took $10^{-2}$ and 500 as typical values of $r_{\min }$ and $r_{\max }$.

In the vicinity of the line heat source, the velocity of the fluid is dominated by the buoyancy forces; thus, it is directed upwards and has a logarithmic singularity. So, for $\gamma$ close to $\pi$, when the forcing flow is downwards, one or two regions of recirculation appear near the line source for all values of $V_{\infty}$. A recirculation zone above a cylinder of finite radius was observed by Badr \& Pop (1988), who studied the symmetric cases $\gamma=\pi$ and $\gamma=0$. The numerical calculations have indicated that the size of the recirculation region depends strongly on $V_{\infty}$ and the direction, $\gamma$, of the free-stream velocity. As an illustration of how the flow features depend on $\gamma$, we show in figures 4 and $5(a-d)$ the streamlines and isotherms, for $V_{\infty}=0.1$ and different values of $\gamma$. The streamlines bounding the recirculation regions are shown with thick lines. One can see the fast disappearance of the recirculation regions when $\gamma$ deviates from $\pi$. For some values of $\gamma$, as in the case $\gamma=0.85 \pi$ represented in figure $5(b)$, only one recirculation region remains. The upper and lower bounds of the recirculation region are shown in figure 6 , as a function of $V_{\infty}$ for $\gamma=\pi$.

Shown in figure 7 are the values of the constant $A_{0}$, appearing in the near source description (2.20) for the temperature, as a function of $V_{\infty}$ and various values of the angle $\gamma$. The upper and lower solid curves, which correspond to $\gamma=\pi$ and $\gamma=0$ respectively, bound the curves resulting from the numerical calculations for intermediate values of $\gamma$; as an example, the curve obtained for $\gamma=\pi / 2$ is shown with circles. For large values of $V_{\infty}$ the flow is governed by forced convection everywhere, and $A_{0}$ tends to the asymptotic expression, shown in figure 7 with a dashed line, $A_{0}=\ln \left(4 / V_{\infty}\right)-\gamma_{E}$, where $\gamma_{E}=0.577 \ldots$ is Euler's constant. Observe the fast approach, with increasing values of $V_{\infty}$, to this asymptotic behaviour, and the small differences when $V_{\infty}$ is of order unity.

The flow field structure for small, non-zero, values of $V_{\infty}$ is, as seen in figure $5(a-d)$, fairly complicated and difficult to describe numerically, because we encounter other scales in addition to $l_{h}$. Even though we do not attempt to give a detailed description 
(a)

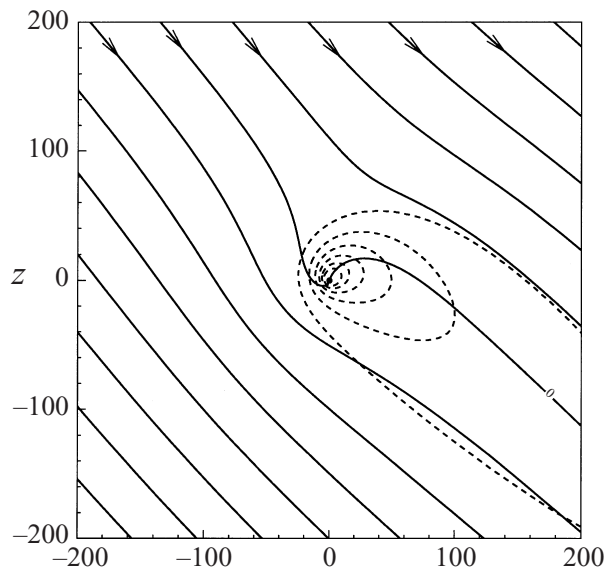

(c)

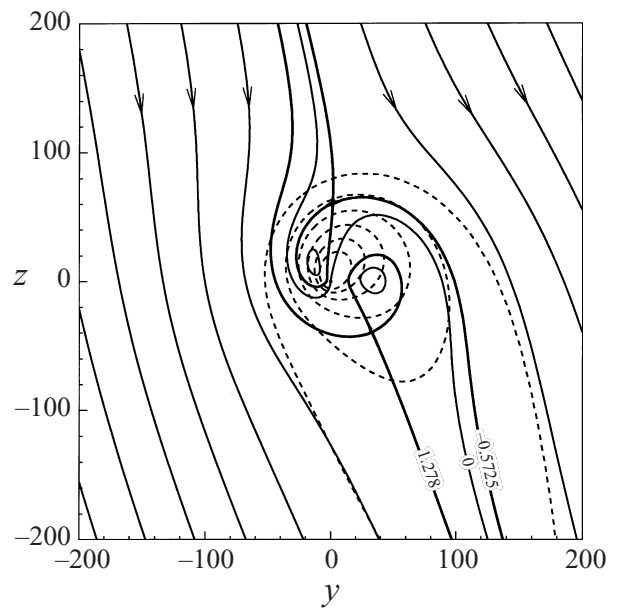

(b)

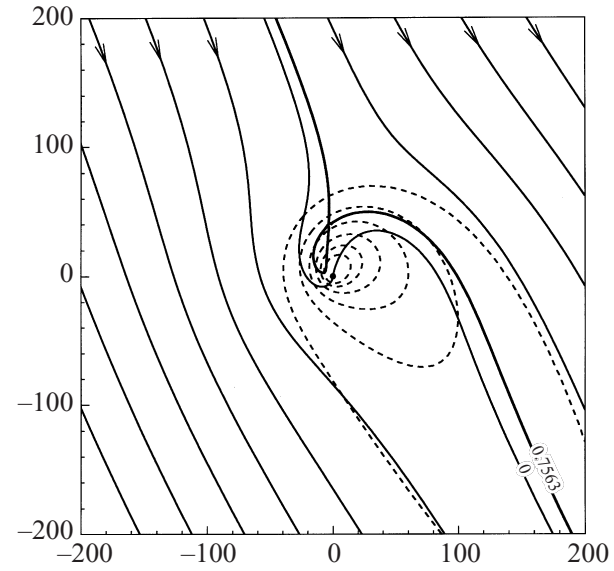

(d)

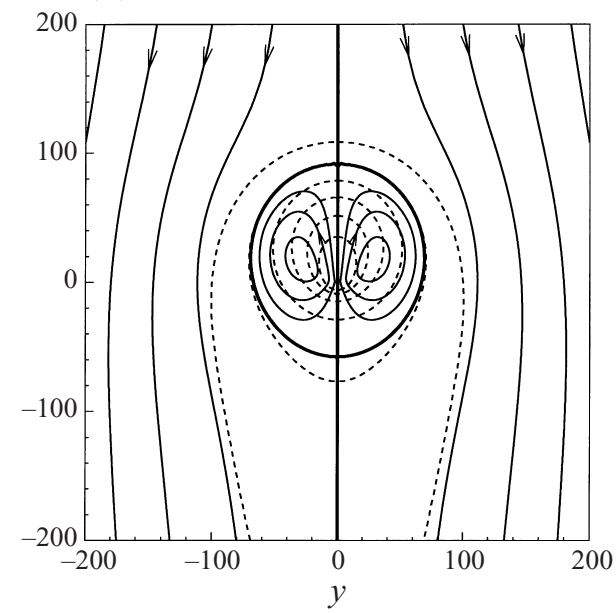

FiguRE 5. Computed flow field around a line source of heat with natural and forced convection with $V_{\infty}=0.1$. The streamlines are shown with solid lines ( $\psi$ at intervals 4 outside the recirculation regions, and 0.5 inside) and the isotherms with dashed lines $\left(\theta\right.$ at intervals $0.05, \theta_{\min }=0.05$ and $\left.\theta_{\max }=0.3\right):(a) \gamma=0.75 \pi,(b) \gamma=0.85 \pi,(c) \gamma=0.9 \pi$ and $(d) \gamma=\pi$. The streamlines bounding the recirculation regions are shown with thick lines.

of the asymptotic structure of the solution for $V_{\infty} \ll 1$, we can indicate that for $r$ (scaled with $l_{h}$ ) of order unity free convection dominates, generating a thermal plume above the source for $r \gg 1$. This plume is bent towards the free stream at distances $r \sim r_{c}=V_{\infty}^{-3 / 2}$, where the entrainment velocities due to the plume, of order $r^{-2 / 3}$, decrease to values of order $V_{\infty}$. For non-horizontal free streams, the longitudinal velocity in the plume is, at $r \sim r_{c}$, still large compared with $V_{\infty}$, so that the two velocities are comparable only at distances large compared with $r_{c}$.

\section{The combined free and forced convection around horizontal heated cylinders in porous media}

We shall consider a horizontal heated circular cylinder of radius $a$ embedded in a porous medium, where, far from the cylinder, the fluid has a temperature $T_{\infty}$ and a 


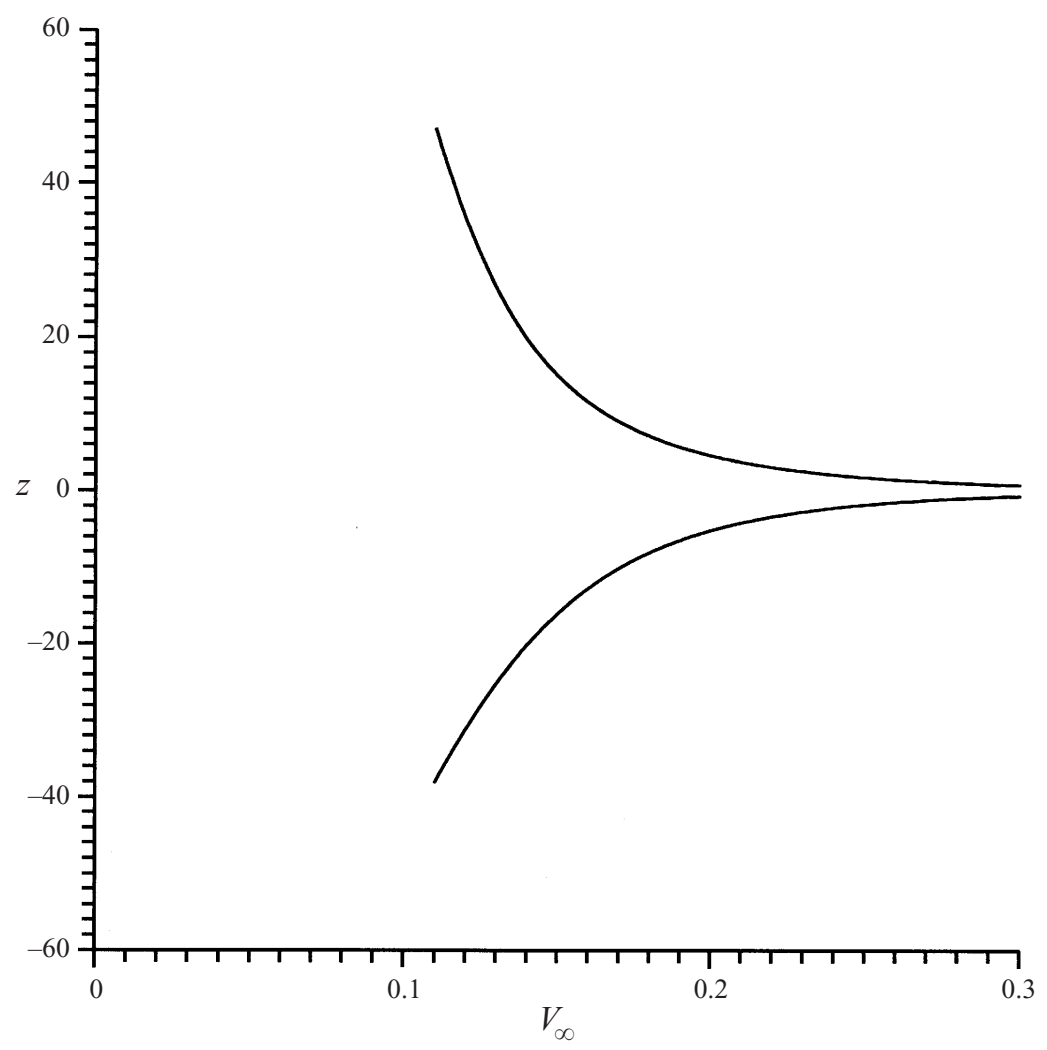

FIGURE 6. The upper and lower values of the coordinate $z$ of the recirculation region around a line source of heat as a function of non-dimensional velocity, for $\gamma=\pi$.

velocity $U_{\infty}$, inclined an angle $\gamma$ with the vertical. Our purpose is to calculate the rate $q$ of heat transfer to the porous medium, per unit length of the cylinder, in terms of its uniform surface temperature $T_{w}$.

Because the far field is determined by $U_{\infty}$ and $q$, independently of the size and shape of the cylinder, it is convenient to pose, for the moment, the problem as to calculate $T_{w}$ as a function of $q$. Then, we shall again use the scales (2.6) for the formulation of the problem, as solving the system of equations (2.7)-(2.8) with the boundary conditions (4.1) at $r \rightarrow \infty$, leading to the asymptotic form given by (2.12) and (2.19). At the circular cylinder surface we use the conditions

$$
\theta=\theta_{w}, \quad \psi=0, \quad \int_{0}^{2 \pi} r \frac{\partial \theta}{\partial r} \mathrm{~d} \varphi=-1 \quad \text { at } \quad r=\varepsilon=a / l_{h} .
$$

Here the surface temperature, $\theta_{w}=\left(T_{w}-T_{\infty}\right) \lambda / q$, has to be determined as a function of $\varepsilon, \gamma$ and $V_{\infty}=U_{\infty} / v_{h}$, which are the parameters remaining in the problem.

The Nusselt number, defined in (3.3), is given by $N u=1 / 2 \pi \theta_{w}$. The Rayleigh and the Péclet numbers,

$$
R a=K \rho_{f} g \beta\left(T_{w}-T_{\infty}\right) a / \alpha \mu_{f} \quad \text { and } \quad P e=U_{\infty} a / \alpha,
$$

are given in terms of the above parameters by

$$
R a / \varepsilon=\theta_{w}=1 / 2 \pi N u, \quad P e / \varepsilon=V_{\infty}
$$




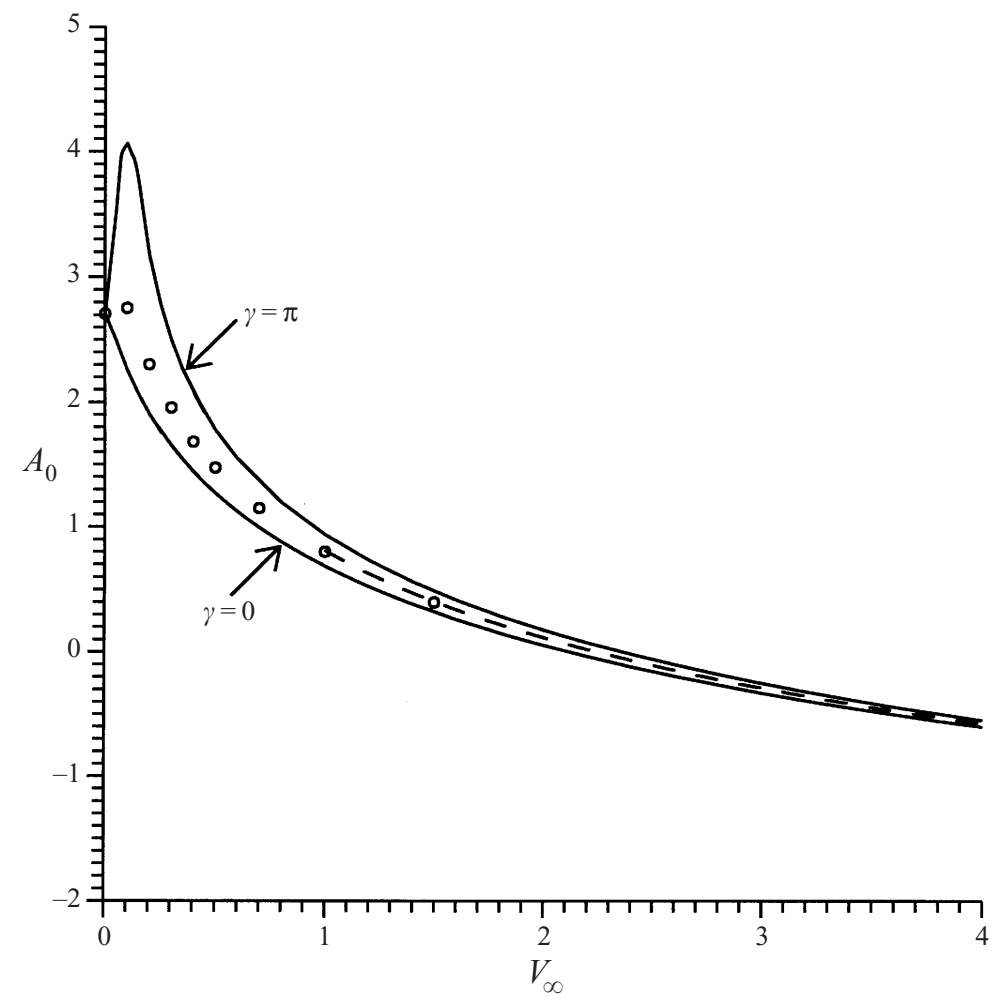

FIGURE 7. Calculated values of $A_{0}$ for $\gamma=0$ (lower solid line), $\gamma=\pi$ (upper solid line) and $\gamma=\pi / 2$ (circles) in the forced and natural convection flow around heated line source. Dashed line: the asymptotic relation $A_{0}=\ln \left(4 / V_{\infty}\right)-\gamma_{E}$, for $V_{\infty} \gg 1$.

so that $N u$ can also be determined as a function of $\gamma, R a$ and $P e$. An example of the form of the isotherms and streamlines is shown in figure 8 for $\varepsilon=1, V_{\infty}=0.1$ and $\gamma=\pi / 2$. The effects of the circulation produced by the jump in pressure across the wake appear clearly in this figure and involve a much slower decay of the velocity perturbations than those associated with the dipole corresponding to the basic irrotational flow around the cylinder.

For $R a \ll 1$ and $V_{\infty} \sim 1$ we can again use, as in $\S 3$, the technique of matched asymptotic expansions to describe the temperature and flow field around the cylinder. In the inner region, $r \sim \varepsilon$, the temperature is given by the heat conduction solution (3.5) In the outer region $\theta$ and $\psi$ are given by the solution of the line source problem, leading for $r \rightarrow 0$ to

$$
2 \pi \theta=-\ln r+A_{0}\left(V_{\infty}, \gamma\right)
$$

The matching condition between (3.5) and (5.4) leads again to the relation

$$
1 / N u=-\ln (2 \pi N u R a)+A_{0}\left(V_{\infty}, \gamma\right),
$$

valid for $R a \ll 1$ and $P e \ll 1$. This relation, together with (5.3), allows us to calculate $N u$ in terms of $R a, P e$ and $\gamma$. In (5.5) $A_{0}=2.71$ for $V_{\infty}=P e / 2 \pi N u R a=0$, and $A_{0}=\ln \left(4 / V_{\infty}\right)-0.577$ for $V_{\infty} \gg 1$, when the heat transfer is dominated by forced convection. So that, for $V_{\infty} \gg 1$ and $P e \ll 1$

$$
1 / N u=-\ln (P e / 4)-0.577 \ldots
$$




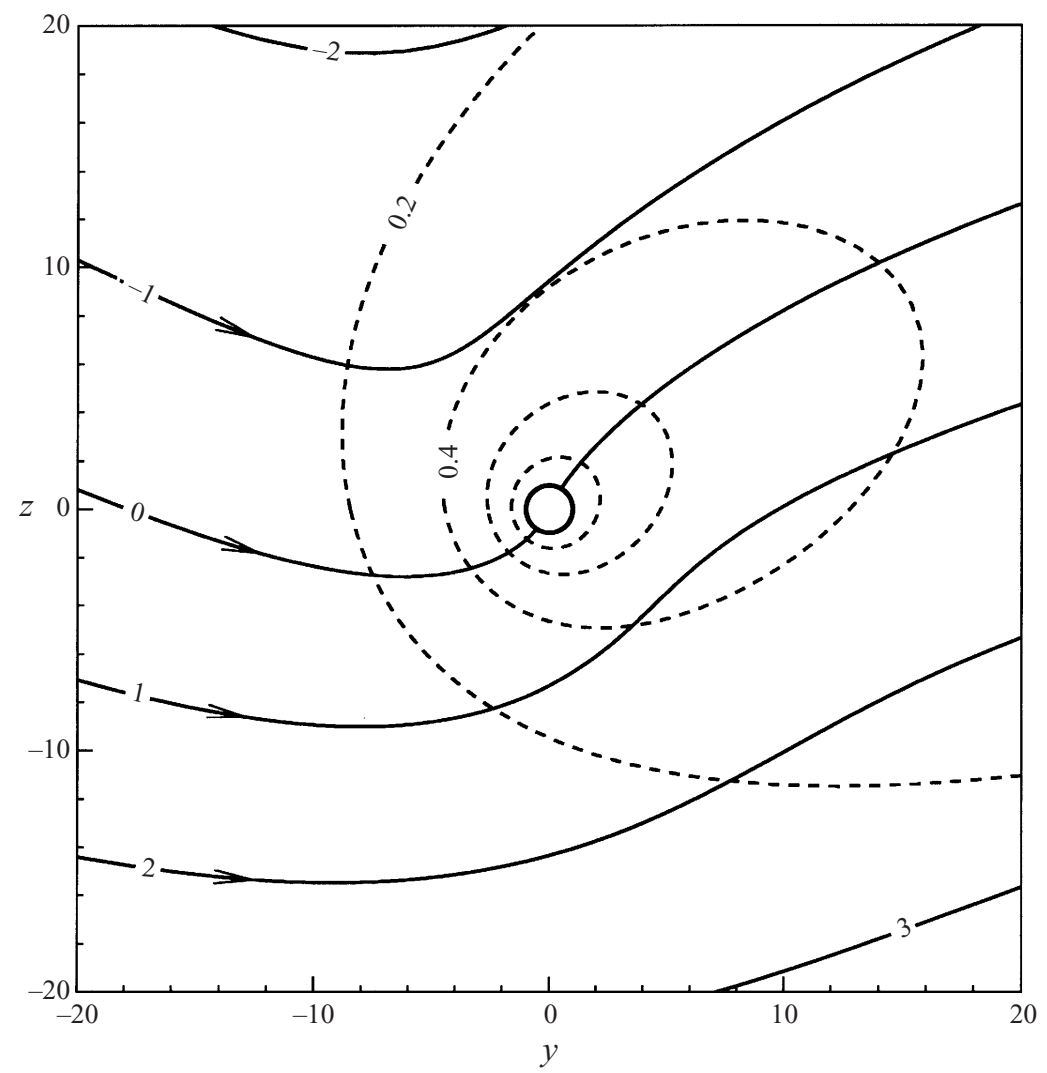

FIGURE 8. Calculated streamlines (solid lines) and isotherms (dashed lines) for the combined natural and (horizontal) forced flow around a heated cylinder with $\varepsilon=1, V_{\infty}=0.1$ and $\gamma=\pi / 2$. To be compared with the case $\varepsilon=0$ shown in figure 4 .

For the description of the heat transfer in pure forced convection, we can use for the velocity field the irrotational flow, without circulation, around the cylinder. The energy equation can be easily solved numerically to evaluate the Nusselt number as a function of the Péclet number. We propose a correlation of the numerical results, accounting for the asymptotic form (5.6) for $P e \ll 1$, and the well known form $N u=0.718 P e^{1 / 2}$ for large Péclet numbers. The resulting expression is

$$
P e / 4=\left(1+b_{1} N u^{1 / 2}+b_{2} N u+b_{3} N u^{2}\right) \exp (-0.577-1 / N u),
$$

where $b_{3}=0.863$, and the adjustable parameters $b_{1}$ and $b_{2}$ are chosen as -0.401 and 0.786 , respectively. This correlation is also plotted in figure 3 , together with the asymptotic description (5.6) for $P e \ll 1$. It is interesting that the numerical results for $\mathrm{Nu}(\mathrm{Pe})$ and $V_{\infty}=1$ cannot be distinguished in figure 3 from the asymptotic results corresponding to pure forced convection. Similarly, the numerical results for $N u(R a)$ and $V_{\infty}=0.1$ are close to the limiting results for $N u(R a)$, corresponding to $V_{\infty}=0$, for moderately small values of $R a$.

In figure 9 we sketch, for $\gamma=\pi / 2$, in the parameter plane $P e, R a$ the lines corresponding to $N u=0.3$ and 1, which follow the numerical results represented by circles. The line $N u=0.3$ has also been calculated using the asymptotic relation (5.5) for small values of $P e$ and $R a$, and it is represented with a dotted line in figure 9. In the figure we also give the asymptotic values corresponding to the limiting cases 


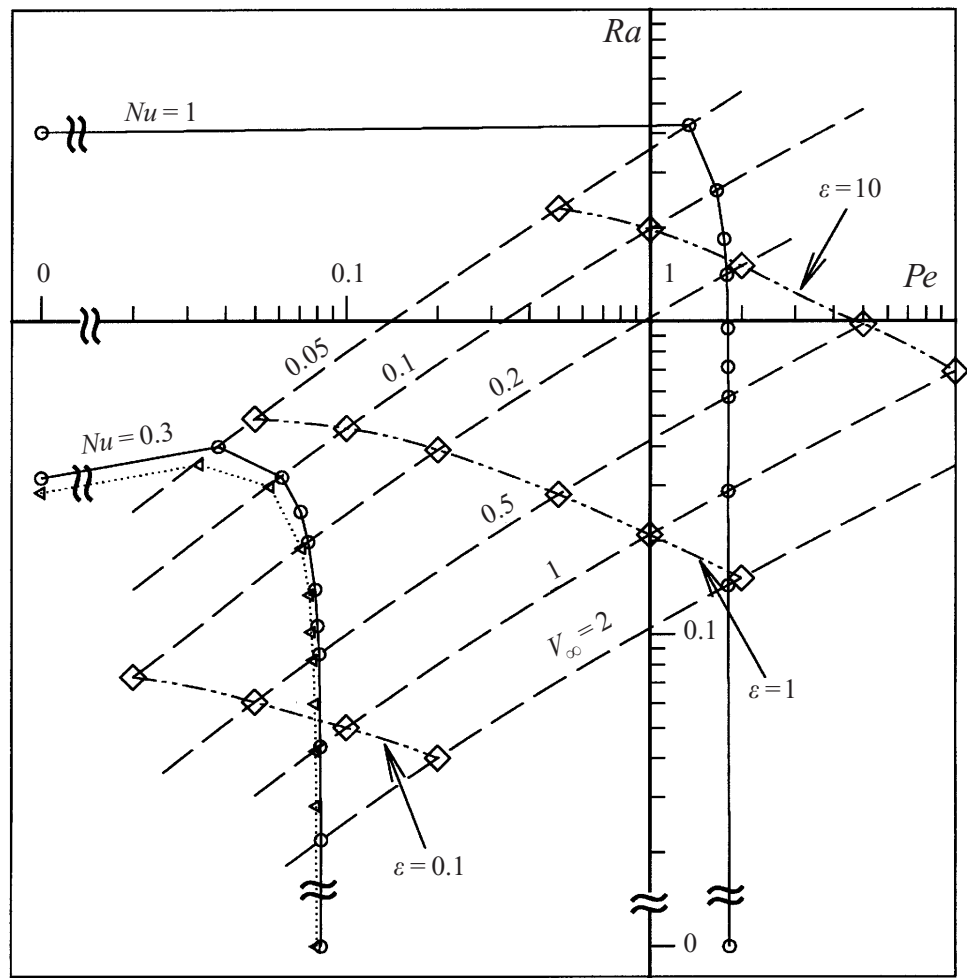

FIGURE 9. Sketch, for combined convection in the parameter plane $P e, R a$, of the lines (solid) of constant $N u=0.3$ and 1 , resulting from the calculated values for $\gamma=\pi / 2$ shown with circles; the dotted line and triangles correspond to the asymptotic relation (5.5) for $N u=0.3$. Also shown are lines of constant $V_{\infty}$ (dashed) and constant $\varepsilon$ (dash-dotted).

$R a \rightarrow 0$ and $P e \rightarrow 0$. Also plotted in the figure are lines corresponding to various constant values of $V_{\infty}$; the values of the Nusselt number can be calculated along these lines using the definitions (5.3). For more information we also give the lines where $\varepsilon$ is $0.1,1$ and 10 .

\section{Concluding remarks}

We began, in $\S 2$ of this paper, with the numerical solution of the problem of free convection due to a horizontal line source of heat in an unbounded saturated porous medium. The description is universal because no parameters are left in the non-dimensional formulation of the problem, when using the scales defined in (2.6). When these scales are used in the formulation of the problem of free convection around circular cylinders only one parameter remains, the non-dimensional cylinder radius $\varepsilon=a / l_{h}$. The numerical solution of the problem has been carried out for a wide range of values of $\varepsilon$, thus obtaining a parametric representation of the relation between the Nusselt and Rayleigh numbers.

For $\varepsilon \ll 1$ we have used the method of matched asymptotic expansions to obtain an explicit relation

$$
R a=(1 / 2 \pi N u) \exp (2.71-1 / N u)
$$

between $R a$ and $N u$ valid for $R a \ll 1$. This was used as a basis to obtain a correlation given by (3.9) and (3.10), for the relation $N u=N u(R a)$. 
A numerical description is also given in $\S 4$ for the flow resulting from the combined effects of forced and natural convection around a line heat source. The results depend on the angle $\gamma$ of inclination with the vertical of the free-stream velocity and on its value, $V_{\infty}$, measured with the characteristic velocity $v_{h}$ due to pure free convection. When the forced flow is directed downwards recirculation regions around the line heat source are observed. The far field includes a thermal wake aligned with the forced flow, where the velocity and temperature perturbations decay as $r^{-1 / 2}$, and an outer irrotational flow, decreasing as $r^{-1}$, represented by a sink and a vortex placed at the line source. This far-field description, due to slow decay with $r$ of the velocity perturbations, is essential for the numerical analysis of the flow and temperature fields around the source.

This far-field description, depending on $V_{\infty}$ and $\gamma$, is also applicable to the forced flow around heated cylinders, even though the near-field description to be obtained numerically depends also on the Rayleigh number. For $R a \ll 1$ the results of the line source analysis have been used to derive a relation (5.5) for the Nusselt number. The values of the Nusselt number resulting from our numerical analysis of the flow around horizontal heated cylinders in porous media can be obtained from figure 9 , where lines of constant $N u, \varepsilon$ and $V_{\infty}$ are shown in the parameter plane $P e, R a$, for $\gamma=\pi / 2$.

Although our numerical calculations have only been carried out for cylinders of circular shape, the asymptotic analysis for small values of the Rayleigh and Péclet numbers can be easily extended to deal with other cylinder shapes. This is so because the outer region is described by the universal line heat source analysis. In the inner region, where heat conduction is dominant, $T$ is no longer given by (3.5). However, complex transform methods can be used to calculate, in terms of $q$, the temperature distribution, $T-T_{w}$, near the cylinder, and the value of the effective radius $a_{e}$ that appears in the temperature distribution in the intermediate matching region, where $\theta$ is of the form (3.5) with $a$ replaced by $a_{e}$. For example, for cylinders of elliptical shape $a_{e}=(a+b) / 2$, in terms of the semi-axes $a$ and $b$.

We do not attempt to give here a description of the asymptotic structure of the flow for large values of $R a$ and $P e$, when $V_{\infty}=P e / R a$ is of order unity and $\gamma \neq 0$. In this case, $\varepsilon \gg 1$ and we might expect the heated region surrounding the cylinder to become a thin boundary layer. This is so for the case $\gamma=0$, analysed by Cheng (1982) with the boundary layer approximation. However, when $\gamma \neq 0$, the boundary layer equations, solved with the condition that the outer flow is dominated by the forced flow, may lead to recirculation and separation and then fail to represent the flow, as shown clearly by the numerical results of Badr \& Pop (1988) for the case $\gamma=\pi$; large recirculation regions are then encountered around the cylinder. We did not aim to include in the paper an stability analysis of the flow.

This research has been supported by the Spanish DCICYT, under Contract No PB94-0400, and by INTA, under Contract No 4070-0036/1996.

\section{REFERENCES}

AfZAL, N. 1985 Two-dimensional buoyant plume in porous media: higher-order effects. Intl J. Heat Mass Transfer, 228, 2029-2041.

BADR, H. M. \& POP, I. 1988 Combined convection from an isothermal horizontal rod buried in a porous medium. Intl J. Heat Mass Transfer 31, 2527-2541.

BEJAN, A. 1978 Natural convection in an infinite porous medium with a concentrated heat source. J. Fluid Mech. 89, 97-107. 
Chang, I. D. 1961 Navier-Stokes solutions at large distances from a finite body. J. Math. Mech. 10, 811-876.

Cheng, P. 1982 Mixed convection about a horizontal cylinder and a sphere in a fluid-saturated porous medium. Intl J. Heat Mass Transfer 25, 1245-1247.

FAROUK, B. \& SHAYER, H. 1988 Natural convection around a heated cylinder in a saturated porous medium. Trans. ASME C: J. Heat Transfer 110, 642-648.

Gebhart, B., Jaluria, Y., Mahajan, R. L. \& Sammakia, B. 1988 Buoyancy-Induced Flows and Transport. Springer.

Hickox, C. E. \& WatTs, H. A. 1980 Steady thermal convection from a concentrated source in a porous medium. Trans. ASME C: J. Heat Transfer 102, 248-255.

Himasekhar, K. \& BaU, H. H. 1988 Thermal convection around a heat source embedded in a box containing a saturated porous medium. Trans. ASME C: J. Heat Transfer 110, 649-654.

Huang, M. J., Yih, K. A., Chou, Y. L. \& Chen, C. K. 1986 Mixed convection over a horizontal cylinder or a sphere embedded in a saturated porous medium. Trans. ASME C: J. Heat Transfer 108, 469-471.

Ingham, D. B. \& Pop, I. 1987 Natural convection about a heated horizontal cylinder in a porous medium. J. Fluid Mech. 184, 157-181.

Kaplun, S. \& Lagerstrom, P. A. 1957 Asymptotic expansions of Navier-Stokes solutions for small Reynolds numbers. J. Math. Mech. 6, 585-593.

KuRdYumov, V. N. \& Liñán, A. 1999 Free convection from a point source of heat, and heat transfer from spheres at small Grashof numbers. Intl J. Heat Mass Transfer 42, 3849-3860.

Lagerstrom, P. A. 1996 Laminar Flow Theory, pp. 34-38. Princeton University Press.

Landau, L. D. \& Lifshitz, E. M. 1987 Fluid Mechanics, §21, Second Edn. Pergamon.

LiÑán, A. \& KuRdYumov, V. N. 1998 Free convection induced by a line heat source, and heat transfer from wires at small Grashof numbers. J. Fluid Mech. 362, 199-227.

Mahony, J. J. 1957 Heat transfer at small Grashof numbers. Proc. R. Soc. Lond. A 238, 412-423.

Merkin, J. H. 1979 Free convection boundary layers on axisymmetric and two-dimensional bodies of arbitrary shape in a saturated porous medium. Intl J. Heat Mass Transfer 22, 1461-1462.

Nield, D. A. \& Bejan, A. 1992 Convection in Porous Media. Springer.

Nield, D. A. \& White, S. P. 1982 Natural convection in an infinite porous medium produced by a line heat source. In Mathematics and Models in Engineering Science (ed. A. McNabb et al.). Dept. Sci. Indust. Res., Wellington, New Zealand.

Van Dyke, M. 1975 Perturbation Methods in Fluid Mechanics, pp. 37-42, Second Edn. Parabolic, Alto CA.

VyNNYCKY, M. \& PoP, I. 1997 Mixed convection due to a finite horizontal flat plate embedded in a porous medium. J. Fluid Mech. 351, 359-378.

Wesseling, P. 1975 An asymptotic solution for slightly buoyant laminar plumes. J. Fluid Mech. 70, $81-87$.

Wooding, R. A. 1963 Convection in a saturated porous medium at large Rayleigh number or Péclet number. J. Fluid Mech. 15, 527-544. 